\title{
The Ethical Use of Placebo in Pediatric Research
}

\author{
Rita A. Gómez-Díaz ${ }^{1 *}$, Niels Wacher ${ }^{1}$, Susana Castañón¹, Carlos A. Aguilar-Salinas², Susan Drier-Jonas ${ }^{1}$ and Alberto Lifshitz-Güinzberg ${ }^{3}$ \\ 'Unidad de Investigación Médica en Epidemiología Clínica, UMAE Hospital de Especialidades Mexico \\ ${ }^{2}$ Departmento de Endocrinología y Metabolismo, Instituto Nacional de Ciencias Médicas y Nutrición Mexico \\ ${ }^{3}$ Jefe de la Unidad de Educación, Investigación y Políticas de Salud, Dirección de Prestaciones Médicas Centro Médico Nacional Siglo XXI, Instituto Mexicano del \\ Seguro Social (IMSS), Mexico
}

\begin{abstract}
The use of placebo remains one of the more hotly debated elements of this facet of medicine. Therefore, the objective of this document is to raise some reflections about its use in children. The World Medical Association, Nuremberg Code, and, in many countries, local regulations consider the principles of beneficence and justice, which consist in offering the best therapies available to children, as long as they are previously evaluated, regardless of race, economic level or social status. However, the special ethical dilemmas of children are not adequately addressed. This article suggests a list of considerations for the use of children in placebo-controlled trials, recommends ethical guidelines, and offers an algorithm based on that proposed for adults for evaluating the ethics of a placebo-controlled trial.
\end{abstract}

Keywords: Placebo; children; vulnerable groups; Declaration of Helsinki; developing countries

\section{Introduction}

The use of placebo is a standard component of medical research. However, some of its indications remain controversial to this day. The World Medical Association [1] Nuremberg Proceedings [2] and, in many countries, local regulations consider the principles of nonmalfeasance and justice (defined as the ethical obligation to distribute the benefits and burdens of research fairly), which consist in offering the best therapies available to children, as long as they are previously evaluated, regardless of race, economic level or social status.

Ethical resolutions in the health field are often taken making decisions for those that cannot, or are limited in, their capacity for decisions, for instance: children, psychiatric patients, elderly, unconscious patients, embryos and fetuses. However, both the physician and the caregivers and their families are under duress and often make decisions based on unrealistic expectations or misunderstandings.

Medical practice in children is quite different than in adults: different doses of medication are required; longer term follow-up, and, more importantly, there remains the risk of long-term adverse events during various stages of growth, development and sexual maturity [3]. A clinical trials.gov search (http://www.clinicaltrials.gov, accessed August 11, 2011) shows 4008 current studies using placebo in children with type 1 diabetes, for example. This increase has led to renewed interest in research ethics issues.

The efficacy of a drug in children, but not the safety and dosage, is usually extrapolated from studies in adults. In addition, the obstacle of age prohibits exercising the principle of autonomy (i.e. the capacity of a person to decide for the more convenient option), along with the parents' implicit anguish demanding the best therapeutic option for their child. In children older than 7 , just as in adults, a consensus is being created to honor the ethical principle of respect for persons, and agreement to take part in a research study is requested by an assent letter after the parents had given their consent [4].

Agreement to participate in a clinical research study is requested with the Informed Consent and/or agreement to a treatment may contrast with the principle of beneficence (i.e. to acknowledge the already known benefits and risks of the treatment), which consists in the best options for the patients whether they are newborns, children or teenagers [5]. In the context of clinical research, beneficence gives rise to the moral obligation to provide research subjects with a reasonable balance of harms and benefits. Establishing what constitutes this balance is one of the central challenges in research ethics oversight [6].

Therefore, the objective of this document is to raise some reflections about the use of placebo-controlled trials in children. At the end of this paper is a proposed algorithm, based on that proposed for adults by RJ Amdur and CJ Biddle, to determine the ethical use of placebocontrolled trials with children [7].

\section{Background and ethical issues about the use of placebo}

The use of placebo in controlled trials has been and continues to be controversial. The 2008 version of the Declaration of Helsinki restricts the use of placebo trials to those where there is no current treatment and there is sound rationale for its use. In such case, the patient receiving placebo, or even no treatment, should not be subject to risk or harm. Placebo may also be used when, for scientifically sound and methodological reasons, such use is necessary to determine the efficacy of an intervention. In addition, the Ethics Committee and any other overseer must take care to avoid abuse of this option [8].

Placebos are commonly used as an inactive treatment to achieve double blinding. Sometimes, placebos are used to offer a "clean" control. The argument is that although a new treatment may be found to be as effective, or more effective, than standard treatment in a clinical

*Corresponding author: Rita A. Gómez Díaz, MD Av. Cuauhtémoc No. 330, Col Doctores. México City 06720, Mexico, Tel: 52-55-5627 6900 Ext 21481; Fax: 5255-10541599; E-mail: ritagomezdiaz@yahoo.com.mx

Received October 14, 2011; Accepted November 15, 2011; Published November 25, 2011

Citation: Gómez-Díaz RA, Wacher N, Castañon S, Aguilar-Salinas CA, DrierJonas S, et al. (2011) The Ethical Use of Placebo in Pediatric Research. J Clinic Res Bioeth 2:120. doi:10.4172/2155-9627.1000120

Copyright: (c) 2011 Gómez-Díaz RA, et al. This is an open-access article distributed under the terms of the Creative Commons Attribution License, which permits unrestricted use, distribution, and reproduction in any medium, provided the original author and source are credited. 
trial, both treatments may very well be ineffective. Investigators need to know the anticipated effects of the control intervention. It has been argued that placebos are the ideal choice to evaluate the efficacy and safety of a drug, as their anticipated benefits are known to be marginal. This argument is based on the blinding of the arms of the study, because if the blinding of the placebo arm is not effective then the protection against expectation effects, biased assessment, contamination, and co-intervention are all lost. Because of the importance of the success of blinding, the Consolidated Standards for Reporting of Trials (CONSORT) Group has explicitly incorporated the issue. Section 11(b) of the CONSORT statement states that the success and/or methods of blinding should be reported in the publication [9].

Moreover, the first controlled study with placebo with the purpose of research was carried out in 1899 [10]. Ever since then, studies controlled with placebo have been controversial, especially when it is known that safe and effective treatments are available for the patients randomly assigned to placebo.

Recently a debate from different points of view has arisen. Methodologically, studies controlled with placebo are considered to be necessary, at least when there is high risk of life-threatening or irreversible morbidity associated with current treatment. Another aspect is that if an effective treatment is available, the use of placebo should be prohibited. Instead, the standard care is used as a substitute for placebo. An intermediate issue is to allow studies controlled with placebo when convincing methodological reasons are presented and a strict ethics evaluation is done, which clarifies that the patients receiving placebo will not be exposed to unnecessary harm and the associated risks are minimized. However, since developing countries have different standards and practices than developed ones, it may be difficult to define when and to what degree an effective treatment exists, since developing countries have many restrictions that limit the availability of many treatments [11]

Nevertheless, the placebo control might be considered when no adequate therapy for the disease exists and/or the active therapy has serious side-effects; it should not last too long or inflict unacceptable risks on the patients, who should be adequately informed and informed consent given [12].

Placebo seems to function in cases where the risks are minimum (e.g.: in studies for the treatment of allergic rhinitis); that is why the Canadian Institutes of Health Research, reached the following conclusion: "The best proof that the inclusion of a placebo in a study is adequate, will be the decision of the patient to participate in agreement that the physician's request is based on a state of moral equilibrium and clinical justification" [13].

The elements of the intervention for the control group should be the nonspecific aspects of the intervention (eg, the benefits of getting a placebo), while the experimental group experiences both non-specific (e.g., the benefits of the placebo) and specific aspects (the benefits of the active intervention). Only in this way can differences between groups be attributed to the specific aspect [14]

\section{Placebo in clinical trials: specific issues for children}

Children are a vulnerable population with the same rights to health as adults and must be protected from undue inclusion in clinical research studies, but they have the right to be included in studies seeking further treatments specific to them. Children require agespecific studies, and, as they are in a state of hormonal change, said studies should guarantee longer follow-up, whether direct or indirect, to discover long-term effects of interventions. In this case, placebocontrolled trials of possible treatments are desirable. As noted in a meta-analysis by Xunrong Luo et. al., clinical trial outcomes frequently differ from pre-trial results [15].

Nevertheless, children pose special considerations. As minors, they are subject to the decisions of the parents. In developing countries, this may mean illiterate or poorly educated people under stress due to their child's condition. Many of them will agree to any suggestion by healthcare staff, including participation in trials [16]. Children with chronic conditions are often subject to unknown long-term risks that may not be taken into consideration in a relatively short-term trial. Some of these factors may not manifest themselves until the child has matured into adulthood.

These characteristics force analysis of clinical research studies in children from a different perspective. Because children and their parents are vulnerable for trials that investigate therapies for diseases affecting minors, the scientific and ethical concerns related to choosing appropriate controls are particularly significant. Unanswered questions include:

1. Definitions of the pediatric populations in which placebo is acceptable

\section{Type of research in which placebo use could be considered}

\section{Definitions of both risks and benefits}

\section{Short and long term effects}

At the end of this paper is a proposed algorithm, based on that proposed for adults by RJ Amdur and CJ Biddle, to determine the ethical use of placebo-controlled trials with children [7].

With all the aforementioned facts, it is necessary to rethink investigations carried out in pediatric populations, since they must reflect safe and strict scientific principles. In addition, the ethics review boards which make vital decisions and have the responsibility of monitoring these trials must be constantly aware of the specifics of the risk-benefit profile and the details of each study, which would necessarily imply advising by specialists in each case, including for the age group involved. There should be ongoing vigilance that the algorithm at the end of this paper is utilized. Some considerations should always be in the mind of the members of the ethics boards when studies involving children and adolescents are under evaluation [17].

Clinical equipoise emerges as the specification of the duty of care between the state and research subjects. It is commonly understood as an ethical requirement emerging from the trust relationship between physician-researchers and patient-subjects and their families. The ethical requirement of clinical equipoise helps to ensure that all research subjects in a trial will receive competent medical care [18].

Finally, the use of unnecessary placebo should be avoided, although it can be acceptable, with safety valves, in carefully outlined situations to help identify the kind of response (high or null). Some examples are:

a) Children and adolescents with depressive disorders, where the response to placebo in a random sample brings the opportunity for those responding to the placebo to avoid the possible risks of active treatment, whereas the opportunity is delayed for those without placebo that are under potentially-effective active medication. In this case the risk is minimal but the benefit noteworthy.

b) In defined periods of time in certain clinical conditions (for instance, the treatment of conditions where a medical emergency is 
rare). Patients with such conditions, including those who were not previously treated, can be reassigned to placebo for a limited period of time as part of the study protocol, provided there are adequate safeguards for the patient's safety.

c) It is ethically safe to stop a pharmacological treatment in stable patients (Obsessive-compulsive disorder, etc.) and have them receive placebo during an established period of time as part of the protocol guidelines as long as safety is adequate.

The Ethical Guidelines of the Council for International Organizations of Medical Sciences [19] for carrying out studies evaluating medications using placebo in pediatric populations are described below:

1) When there is not a commonly-accepted treatment for the condition and the medication under study is the first one that can modify the course of the disease's progression.

2) When the commonly-used treatment for the condition is of questionable efficacy.

3) When the commonly-used treatment for the condition has undesirable side effects and the risks of use can be significantly greater than the benefits.

4) When the placebo is used to identify the incidence and severity of undesirable side effects produced when adding a new treatment to an established regimen, or

5) When the disease is characterized by an increase in frequency, exacerbations or spontaneous remission and the efficacy of the treatment has not been proved.
Together with the above, in a recognized publication [20], some topics are recommended for research in children, using the standards established by the Federal Regulations of the United States, which specify four categories of research, although there is still no reference to the use of placebo in children; there is incongruity in the definition of age for an "infant" patient, and 18 years of age is mentioned as the age of adulthood for some situations while 21 years of age is mentioned for others. In Mexico, the General Health Law establishes an age limit of 16 years, without mentioning special situations.

All of these facts raise consideration of certain details. The national and international organisms in charge of ethical issues in medical practice and research have as their responsibility establishing guidelines, currently weak or non-existent, regarding the use of placebo in the pediatric patient and defining terms with precision, since in the medical literature "placebo" and "control group" can often be found as synonyms, and it is well known that each has a different psychophysiological impact on the patient [21]. There are mixed interpretations of what constitutes a "minimal risk" and a "minor increase over minimal risk". It is generally assumed that "minimal risk" is no greater than the risk faced in daily life [17]. However, the risks faced in daily life can vary considerably. Without strict definitions, it is left to the discretion of the ethics review boards to determine if a trial should proceed or not. In addition, it has been suggested that each intervention, placebo and drug, should be assessed for risk-benefit profile both separately and collectively. Using this criteria, placebocontrolled trials should only be carried out when both the placebo intervention and the drug intervention comply with the guidelines [22].

As a final consideration, it might be useful for studies in pediatric

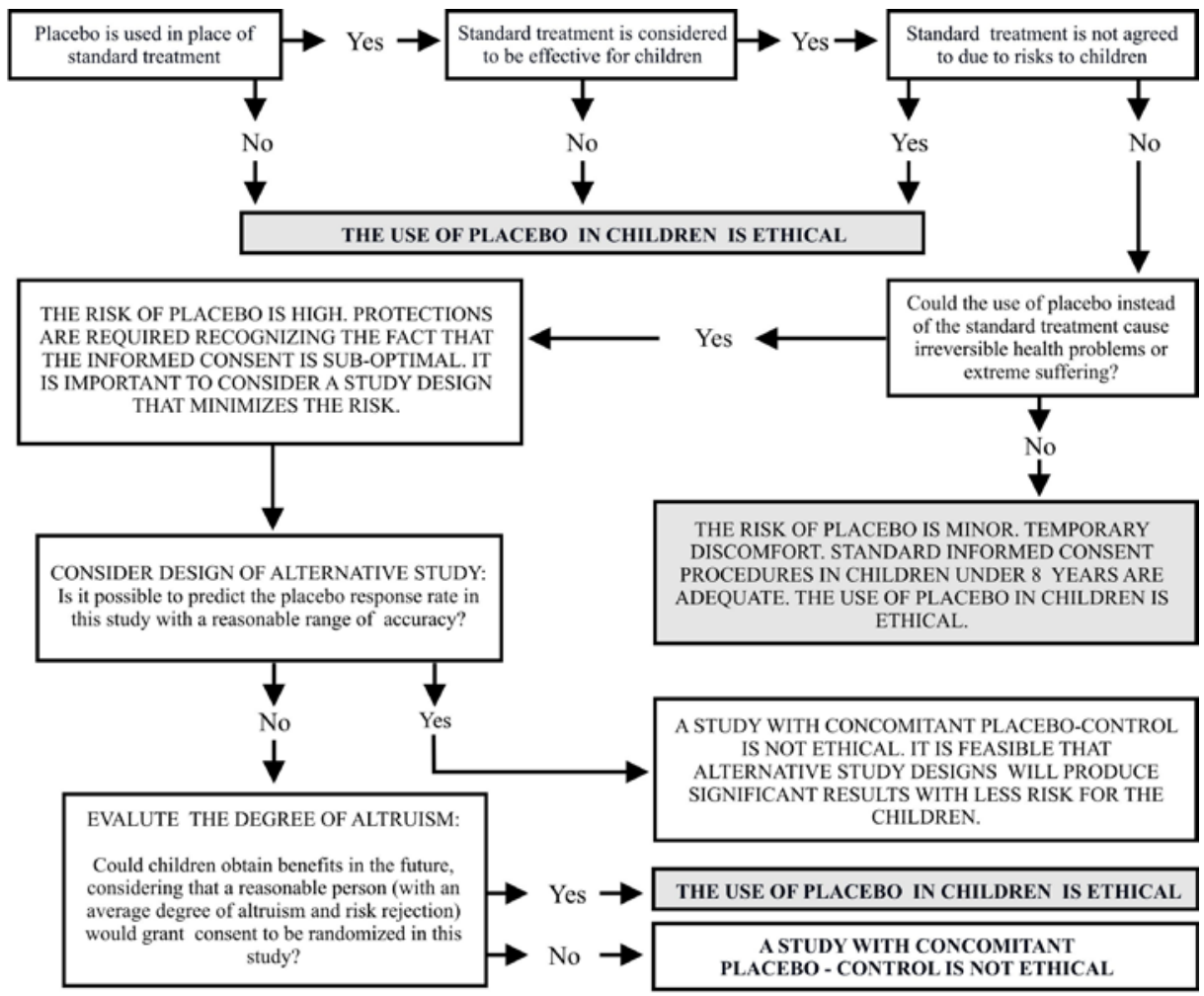

Figure 1: ALGORITHM FOR EVALUATING THE ETHICS OF A PLACEBO CONTROLLED TRIAL IN CHILDREN. (Modified form Amdur RJ, Biddle CJ. Int J Cancer 2001; 96: 261269) 
Citation: Gómez-Díaz RA, Wacher N, Castañon S, Aguilar-Salinas CA, Drier-Jonas S, et al. (2011) The Ethical Use of Placebo in Pediatric Research. J Clinic Res Bioeth 2:120. doi:10.4172/2155-9627.1000120

Page 4 of 4

populations to be guided by a modified algorithm of that which has been validated and used in adults (See Algorithm Figure 1).

The algorithm should be applied according to level of risk and can be used to minimize risks, which might offer some advantages for medical decision-making, and it may also be applied in order to evaluate the ethics of studies with placebo control in minors. Use of this algorithm will help avoid the ill-defined concept of minimum risk in favor of more measureable parameters.

\section{Conclusions}

Every investigator who wants to ethically explore the benefits of new pharmacological treatments in children and adolescents should know the Agreements of The World Medical Association and the most recent revision of the Declaration of Helsinki regarding the use of placebo. It is necessary for an ad hoc team to review and update the pediatric guidelines suggested in the previous paragraphs regarding the use of placebo in clinical trials, taking into consideration the algorithm as modified from adults. Such a group must include professionals having clinical and academic experience: investigators, bio-ethicists, government, and the participation of the pharmaceutical industry, all working for the same purpose. Taking these considerations into account may help clarify the on-going controversy over placebocontrolled trials in children, and will guide researchers and ethics committees to better study design and realization.

\section{References}

1. World Medical Association (2008) Declaration of Helsinki: Ethical Principles for Medical Research involving Human Subjects. [Date accessed: July 17, 2011].

2. Transcripts of the Nuremberg Doctors Trials are archived by The University of Missouri, Kansas City at www.law.umkc.edu/.../ftrials/nuremberg/ nurembergdoctortrial.html 2.[Date accessed: July 17, 2011]

3. Committee on Drugs, 1992-1993. (1995). Guidelines for the Ethical Conduct of Studies to Evaluate Drugs in Pediatric Population. Pediatrics 95: 286-295.

4. Whittle A, Shah S, Wilfond B, Gensler G, Wendler D (2004) IRB practices regarding assent in pediatric research. Pediatrics 113:1747-1752.

5. Diario Oficial de la Federación, 7 de febrero (1984). Estados Unidos Mexicanos, Secretaria de Salubridad y Asistencia. Ley General de Salud.
6. Emanuel E, Menikoff J (2011) Reforming the Regulations Governing Research with Human Subjects. New Engl J Med [Date accessed: July 26, 2011].

7. Amdur R, Biddle C (2001) An algorithm for evaluating the ethics of a placebocontrolled trial. Int J Cancer 96: 261-269.

8. Puri, KS, Suresh KR, Gogtay NJ, Thatte UM (2009) Declaration of Helsinki, 2008: implications for stakeholders in research. J Postgrad Med 55: 131-134.

9. Schulz KF, Altma DG, Moher D for the CONSORT Group. 2010. CONSORT 2010 statement: Updated guidelines for reporting parallel group randomised trials. J Pharmacol Pharmacother 1: 100-107.

10. Cabot R, Whoriskey J (1899) Substitutes for tuberculin as a means of diagnosis. J Boston Soc Med Sci 3: 71-74.

11. Wendler D, Emanuel EJ, Lie RK (2004) The Standard of Care Debate: Can research in developing countries be both ethical and responsive to those countries' health needs? Am J Public Health 94: 923-928.

12. De Deyn P, D’Hooge (1996) Placebos in clinical practice and research. J Med Ethics 22: 140-146.

13. Sibbald B (2002) Canada to reconsider ethics of placebo-controlled trials. Canadian Medical A J 166: 641.

14. Sibinga E, Jennings J (2010) Research and Statistics: Strengths and Limitations of Randomized, Controlled Trials. Pediatr Rev 31: 296-297.

15. Luo X, Herold K, Miller S (2010) Immunotherapy of Type 1 Diabetes - Where are we and Where Should We Be Going. Immunity 32: 488-499.

16. Blodgett LJ, Boyer W, Turk E (2005) "No thank you, not today": Supporting Ethical and Professional Relationships in Large Qualitative Studies [38 paragraphs]. Forum Qualitative Sozialforschung / Forum: Qualitative Socia Research, 6(3), Art 35.

17. 45 CFR 46.102(i)

18. Binik A, Weijer C, Sheehan M (2011) Minimal Risk Remains an Open Question Am J Bioeth 11: 25-27.

19. Council for International Organizations of Medical Sciences (2002) Internationa ethical guidelines for biomedical research involving human subjects. Bull Med Ethics 182: 17-23.

20. Knudson P (2002) Ethical Issues in Humans Subject Research Research with Children. Arch Med Res 33: 203-204.

21. Denny W (1995) The use of placebo controls. N Engl J Med 332: 61-62.

22. Miller F, Wendler D, Wilfond B (2003) When do the federal regulations allow placebo-controlled trials in children? J Pediatr 142: 102-107. 